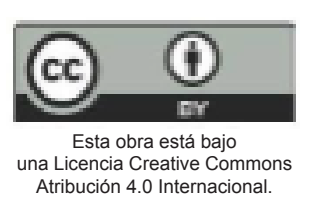

\title{
Empleabilidad de los egresados de Estomatología
}

\author{
Employability of graduates of Stomatology \\ Milagros Carrasco ${ }^{1, \mathrm{~b}}$, Francisco Orejuela ${ }^{1, \mathrm{~b}}$ \\ RESUMEN
}

Objetivo: Evaluar la empleabilidad de los egresados de estomatología en base a la Encuesta Nacional a Egresados Universitarios 2014 del Instituto Nacional de Estadística e Informática. Material y métodos: La investigación fue descriptiva, retrospectiva y transversal. La población del estudio fueron 245 egresados de 33 universidades del país, entre los años 2006 y 2010. Resultados: El 79,6\% de los egresados se encontraban empleados y principalmente como odontólogos (77,3\%), siendo el promedio de horas laboradas de 33,8 horas por semana y la retribución económica de 505,3 soles. E1 33,5\% de los egresados se encontraba en Lima, 68,25\% titulados, 21,2\% con grado de bachiller y el 33,5\% con estudios de posgrado. El 22,2\% tenían otro trabajo, principalmente de odontólogo (66,5\%). El 35,1\% cambió de empleo y el primer empleo tenía relación con la profesión en el 96,5\%. El tiempo que toma conseguir el primer empleo es menor a un mes (40,7\%) y por medio de familiares y amigos (37,2\%). Conclusiones: Los egresados de estomatología adquirieron competencias para la empleabilidad.

PALABRAS CLAVE: Trabajo, capital humano, mercado laboral

\section{SUMMARY}

Objective: To evaluate the employability of the stomatology graduates based on the National Survey of University Graduates 2014 of the National Institute of Statistics and Informatics. Material and methods: The research was descriptive, retrospective and transversal. The study population included 245 graduates from 33 universities in the country, from 2006 to 2010 . Results: $79.6 \%$ of the graduates were employed and mainly as dentists (77.3\%), with an average of 33.8 hours per week worked hours and an economic compensation of 505.3 soles. 33,5\% of the graduates were in Lima, $68.25 \%$ graduates, $21.2 \%$ with a bachelor's degree and $33.5 \%$ with postgraduate studies. $22.2 \%$ had another job, mainly dentistry (66.5\%). 35.1\% changed employment and the first job was related to the profession in $96.5 \%$. The time it takes to get the first job is less than one month (40.7\%) and through family and friends (37,2\%). Conclusions: Stomatology graduates acquired skills for employability.

KEY WORDS: Work, human capital, job market. 


\section{INTRODUCCIÓN}

La noción de empleabilidad puede considerarse como una ampliación y actualización de la Teoría del Capital Humano, la cual considera que la educación es una inversión y que el conocimiento, como la salud, radica en los individuos, el cual es un factor de productividad (1). Los estudios iniciales se remontan al siglo XIX, cuando Max Weber estudió las estrategias para identificar durante la selección de personal, la voluntad para hacer o producir algo, considerando que ésta influye en el desempeño de un trabajador (2).

El término empleabilidad es el resultado de la traducción del término inglés employability como la unión de las palabras employ (empleo) y ability (habilidad) (3). Se define como un conjunto de aptitudes y actitudes que permiten a una persona conseguir, conservar un empleo (4), obtener un nuevo empleo si se requiriese (5), mejorar su trabajo y adaptarse al cambio, elegir otro empleo cuando lo deseen o pierdan el que tenían, integrarse más fácilmente en el mercado de trabajo en diferentes períodos de su vida (6) y de tener éxito en sus puestos, lo cual les beneficia a ellos mismos, a la fuerza de trabajo, a la comunidad y a la economía (7). Es considerada una competencia personal y una cuestión de responsabilidad social, compartida entre individuos, organizaciones e instituciones (8).

La relación de un programa académico con el entorno es a través de los egresados (9) y la información que proporcionan sobre inserción laboral $y$ el contexto en que se desenvuelven debe permitir a las instituciones analizar sus propósitos, así como revisar y actualizar sus planes de estudios, tal como lo plantea el modelo de acreditación de la calidad educativa en el país (10).

El estudio CHEERS, Careers after Higher Education: an European Research Study, sobre la enseñanza superior y empleo de los graduados en once países de Europa y Japón, encontró que el $40 \%$ buscaban empleo antes de graduarse y más del $30 \%$ inmediatamente después de graduarse, siendo el tiempo promedio de búsqueda del primer empleo entre tres y seis meses. Una quinta parte consideraban que utilizaban muy poco o nada los conocimientos $\mathrm{y}$ habilidades adquiridas con los estudios y una sexta parte no veía vínculo alguno entre los estudios cursados y las tareas del trabajo. Para la mayoría, el empleo no les suponía una situación que les decepcionara y al que tuvieran que adaptarse; por el contrario, cuatro años después de graduarse, más del $40 \%$ consideraba que su situación era mejor de la esperada, frente a solo un $20 \%$ que pensaba que era peor (11).

Para el proyecto REFELEX, El Profesional Flexible en la Sociedad del Conocimiento: Nuevas Exigencias en la Educación Superior en Europa, se encuestó a más de 40000 graduados universitarios que obtuvieron la titulación cinco años antes del momento de la entrevista. Se encontró que el 74\% trabajaba en el nivel más alto de las ocupaciones (64\% como profesionales, y $10 \%$ como directivos), proporción similar al del estudio CHEERS. El 81\% trabajaba a tiempo completo, 42 horas semanales en promedio y permanecían en actividades de formación. El nivel de satisfacción en el empleo entre los graduados es alto, el $69 \%$ afirma que se siente satisfecho, y solo el $10 \%$ insatisfecho (12).

El proyecto ALFA PROFLEX, El Profesional Flexible en la Sociedad del Conocimiento, surgió como una iniciativa para Latinoamérica similar al REFLEX para lo cual se encuestó a 9808 graduados de 36 universidades de 10 países (Argentina, Bolivia, Brasil, Chile, Colombia, Honduras, México, Panamá, Puerto Rico y Uruguay) en la que se les preguntó sobre el mundo laboral cinco años después de haber finalizado sus estudios superiores. E1 16,2\% trabaja por cuenta propia y los que laboraban en áreas de Educación y Salud se encontraban más satisfechos que los de Ciencias Sociales, Humanidades o Economía y Empresa. Los graduados conceden mayor importancia a ciertos valores laborales como la oportunidad de aprender cosas nuevas, la estabilidad laboral y las perspectivas profesionales; por el contrario, desplazan el prestigio social al último lugar en su lista de prioridades (13).

Se investigaron a 273 egresados de odontología de una universidad colombiana. La edad promedio fue $27,4 \pm 1,7$ años. El $86.7 \%$ de los egresados ejercía la odontología y la educación que recibieron en la universidad fue calificada como muy buena por el $85.1 \%$. (14), 
En nuestro país, evaluó a 132 egresados de estomatología en una universidad privada. Las edades fluctúan entre 24 y 54 años, predominando el sexo masculino, $18.2 \%$ tenía maestría y más de $65.0 \%$ se mantenía actualizado. El 99.24\%\% ejercía su profesión, $1 / 3$ se dedica a la práctica privada exclusiva y $2 / 3$ realiza práctica privada e institucional. La mayor proporción gana en total menos de 1500 dólares mensuales (15).

En esta investigación se evaluaron algunas variables de empleabilidad de los egresados de estomatología en el Perú, en base a los resultados de la Encuesta Nacional a Egresados Universitarios 2014, realizada por el Instituto Nacional de Estadística e Informática (INEI), órgano rector del Sistema Estadístico Nacional, a través de la Dirección Nacional de Censos y Encuestas, en virtud del Convenio Interinstitucional firmado con el Ministerio de Educación (16).

El objetivo del presente trabajo fue evaluar la empleabilidad de los egresados de estomatología en base a la Encuesta Nacional a Egresados Universitarios 2014 del Instituto Nacional de Estadística e Informática.

\section{MATERIAL Y MÉTODOS}

El estudio fue descriptivo, retrospectivo y transversal. Se empleó como fuente secundaria la Encuesta Nacional a Egresados Universitarios, realizada por el INEI en el año 2014, la cual fue aplicada a egresados de las 100 universidades censadas en el 2010. La población del estudio lo constituyeron 245 egresados de las carreras de estomatología/ odontología, pertenecientes a 33 universidades del país, egresados entre los años 2006 y 2010.

Los participantes del estudio son una submuestra de los egresados seleccionados de manera probabilística, estratificada, de lista y unietápica del marco muestral del II Censo Nacional Universitario 2010, el cual estuvo compuesto de 213210 estudiantes universitarios. Para la selección de los 21142 egresados universitarios, se cumplieron los criterios de al menos un año de egreso, menos de tres años de atraso de estudios en el curso de su carrera y no contar con más de 25 años de edad en el último año de estudios.
El recojo de información fue por medio de Tablet. La base de datos se encuentra en formato SPSS y está dividida en ocho módulos, los cuales contienen grupos de preguntas de la encuesta. Se trabajó con los módulos de Educación ( $\left.\mathrm{N}^{\circ} 590\right)$ y Empleo e Ingresos ( N592).

Mediante tres ítems del cuestionario, la variable empleabilidad fue dimensionada con las subvariables laborar en la profesión odontológica, horas de trabajo y remuneración. Las preguntas eran cerradas

Se obtuvieron las frecuencias y porcentajes para las variables cualitativas, para las variables cuantitativas, promedios y desviación estándar. La información se presentó por medio de gráficas y tablas.

Entre las limitaciones del estudio se encuentra el empleo de un cuestionario con algunas preguntas que no necesariamente eran las más adecuadas a los objetivos del estudio. Por otro lado, no se ha evaluado la influencia de otros factores en la empleabilidad de los egresados.

\section{RESULTADOS}

El 56,7\% de los egresados era del sexo masculino, con edades entre 21 a 30 años. La mayoría proviene de Lima y Arequipa; en contraste, Amazonas, Madre de Dios y Tumbes tuvieron menor porcentaje de egresados (Tabla 1). La mayoría (38,4\%) egresó el año previo al estudio y dos años antes (26,9\%). El 68,2\% había obtenido el título profesional, $21,2 \%$ el grado de bachiller y 10,6\% tenían la condición de egresado. El 33,5\% de los egresados realizó o se encontraba realizando estudios de posgrado, principalmente diplomados. En cuanto al número de diplomados o maestrías que estudiaron o vienen estudiando, en su mayoría era solo uno.

Con respecto a la pregunta, “¿La semana pasada tuvo usted algún trabajo?", 195 (79,6\%) respondieron afirmativamente. Seis $(12,0 \%)$ de los egresados que no trabajaron la semana anterior retornaron al empleo fijo que tenían y cuatro $(9,1 \%)$, al negocio propio.

La ocupación principal de los egresados fue el de odontólogo (77,3\%), como se visualiza en la Tabla 2. El trabajo que realizan está relacionado a su formación profesional en el grupo Dentista auxiliar y ayudante de odontología $(11,1 \%)$. En promedio, laboraban 
Tabla 1. Distribución de egresados según ubicación geográfica

\begin{tabular}{|c|c|c|}
\hline Región & Frecuencia & Porcentaje \\
\hline Amazonas & 1 & 0.4 \\
\hline Ancash & 8 & 3.3 \\
\hline Apurímac & 4 & 1.6 \\
\hline Arequipa & 22 & 9.0 \\
\hline Ayacucho & 9 & 3.7 \\
\hline Cajamarca & 7 & 2.9 \\
\hline Callao & 4 & 1.6 \\
\hline Cusco & 8 & 3.3 \\
\hline Huancavelica & 4 & 1.6 \\
\hline Huánuco & 9 & 3.7 \\
\hline Ica & 4 & 1.6 \\
\hline Junín & 12 & 4.9 \\
\hline La Libertad & 5 & 2.0 \\
\hline Lambayeque & 13 & 5.3 \\
\hline Lima & 82 & 33.5 \\
\hline Loreto & 7 & 2.9 \\
\hline Madre De Dios & 1 & 0.4 \\
\hline Moquegua & 3 & 1.2 \\
\hline Pasco & 2 & 0.8 \\
\hline Piura & 12 & 4.9 \\
\hline Puno & 17 & 6.9 \\
\hline San Martin & 3 & 1.2 \\
\hline Tacna & 4 & 1.6 \\
\hline Tumbes & 1 & 0.4 \\
\hline Ucayali & 3 & 1.2 \\
\hline Total & 245 & 100.0 \\
\hline
\end{tabular}

33,8 horas/semana $(\mathrm{DE}=15,9)$, con una variación de 4 a 75 horas/semana. Un 34,7\% desearía trabajar más horas, en promedio 12,2 horas/semana ( $\mathrm{DE}=6,9)$. El ingreso promedio mensual fue de 505,3 soles $(\mathrm{DE}=$ $1305,7)$.

El 22,2\% tenía otro trabajo, siendo en su mayoría la de odontólogo (Tabla 3). El 35,1\% cambió de empleo con respecto al primer trabajo.

El primer empleo estuvo relacionado a la profesión en $83(96,5 \%)$ de los 86 egresados que cambiaron de empleo. El tiempo promedio que les demandó buscar el primer empleo fue 2,1 meses ( $D S=1,4$ ), siendo menor a un mes en el 40,7\% de los casos (Tabla 4). El $64,9 \%$ no cambió de empleo con respecto al primer trabajo. El 69,8\% tuvo dos o tres empleos desde que egresó (Gráfico 1).

Fueron diversos los medios para conseguir el primer empleo, principalmente por intermedio de familiares y amigos (37,2\%). Ver Gráfico 2.

Los egresados que cambiaron de empleo consideraron que la formación que recibieron de la universidad les ayudó a obtener su primer empleo fueron $75(87,2 \%)$.

\section{DISCUSIÓN}

La empleabilidad de los egresados de estomatología fue evaluada como la condición de 
Tabla 2. Ocupación principal de los egresados

\begin{tabular}{lll}
\hline Ocupación principal & Frecuencia & Porcentaje \\
\hline Odontólogo (cirujanos) & 167 & 77.3 \\
Dentista auxiliares y ayudantes de odontología & 24 & 11.1 \\
Médicos y profesionales afines & 6 & 2.7 \\
Técnicos en radiología y tecnólogos médicos & 1 & 0.5 \\
Técnicos en administración, contabilidad, derecho & 3 & 1.4 \\
Agentes de seguro y ventas & 2 & 0.9 \\
Cantantes & 1 & 0.5 \\
Jefes de empleados administrativos & 1 & 0.5 \\
Empleados de oficina y operadoras de maquinas & 3 & 1.4 \\
Cajeros, pagadores, cobradores de ventanilla & 2 & 0.9 \\
Agricultores (explotadores) & 2 & 0.9 \\
Vendedor de productos de tocador e higiene & 1 & 0.5 \\
Personal de servicios & 2 & 0.9 \\
Obrero de minas & 1 & 0.5 \\
Total & $216^{*}$ & 100.0 \\
\hline
\end{tabular}

*29 datos perdidos

Tabla 3. Ocupación secundaria de los egresados

\begin{tabular}{lll}
\hline Ocupación secundaria & Frecuencia & Porcentaje \\
\hline Odontólogo & 38 & 65.5 \\
Asistente dental & 2 & 3.4 \\
Profesores (universidades/centros de educación) & 2 & 3.4 \\
Administradores de empresas & 2 & 3.4 \\
Asistente administrativo & 2 & 3.4 \\
Publicista & 1 & 1.8 \\
Mozo & 1 & 1.8 \\
Modelo & 1 & 1.8 \\
Vendedor & 4 & 6.9 \\
Preparadora de torta & 2 & 3.4 \\
Personal de servicios & 2 & 3.4 \\
Mensajeros y repartidores & 1 & 1.8 \\
Total & 58 & 100.0 \\
\hline
\end{tabular}

encontrarse laborando en su profesión, número de horas de trabajo y la remuneración que percibe.

La proporción de los que se encontraban trabajando (80\%), puede ser considerado alto si se compara con la tasa de ocupación de $69,7 \%$ reportada en el país en el año 2014 (17). Sin embargo, es inferior a lo reportado por estudios realizados en egresados de estomatología, donde el 99,24\% (15) ejercían su profesión, diferencia que podría estar explicada porque se evaluaron a egresados de los últimos 25 años y en nuestra investigación, los sujetos evaluados tenían entre uno y tres años de egreso. Similarmente, comparado a un estudio realizado en Colombia (14), el porcentaje de los egresados entre 4 y 8 años previo al estudio que se encontraba laborando superaba en siete puntos a nuestro resultado. 
Tabla 4. Tiempo de búsqueda del primer empleo

\begin{tabular}{lll}
\hline Tiempo de búsqueda & Frecuencia & Porcentaje \\
\hline Menos de un mes & 35 & 40,7 \\
Entre 1 a 3 meses & 31 & 36,0 \\
Entre 3 a 6 meses & 11 & 12,8 \\
Entre 6 meses a menos de un año & 1 & 1,2 \\
Entre uno a dos años & 1 & 1,2 \\
No buscó empleo & 7 & 8,1 \\
Total & 86 & 100,0 \\
\hline
\end{tabular}

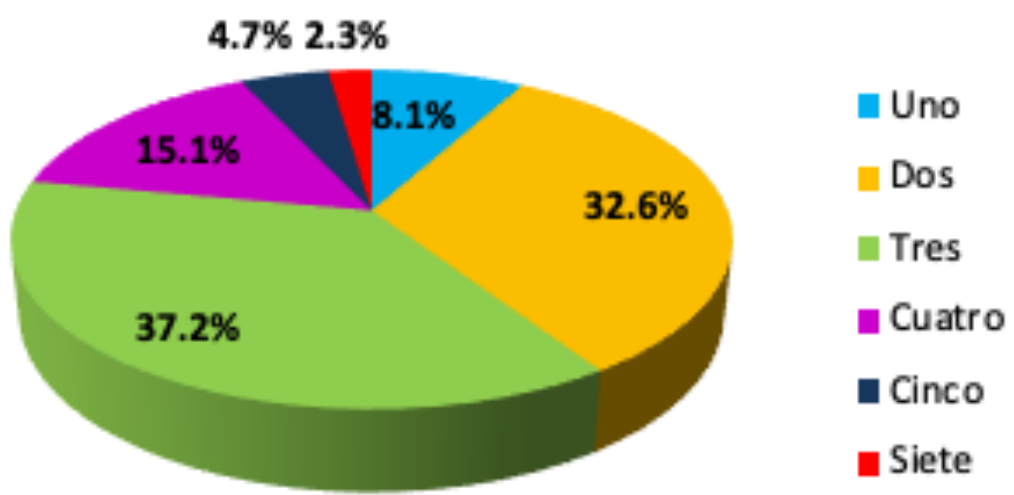

Gráfico 1. Porcentaje de egresados según número de empleos desde el egreso.

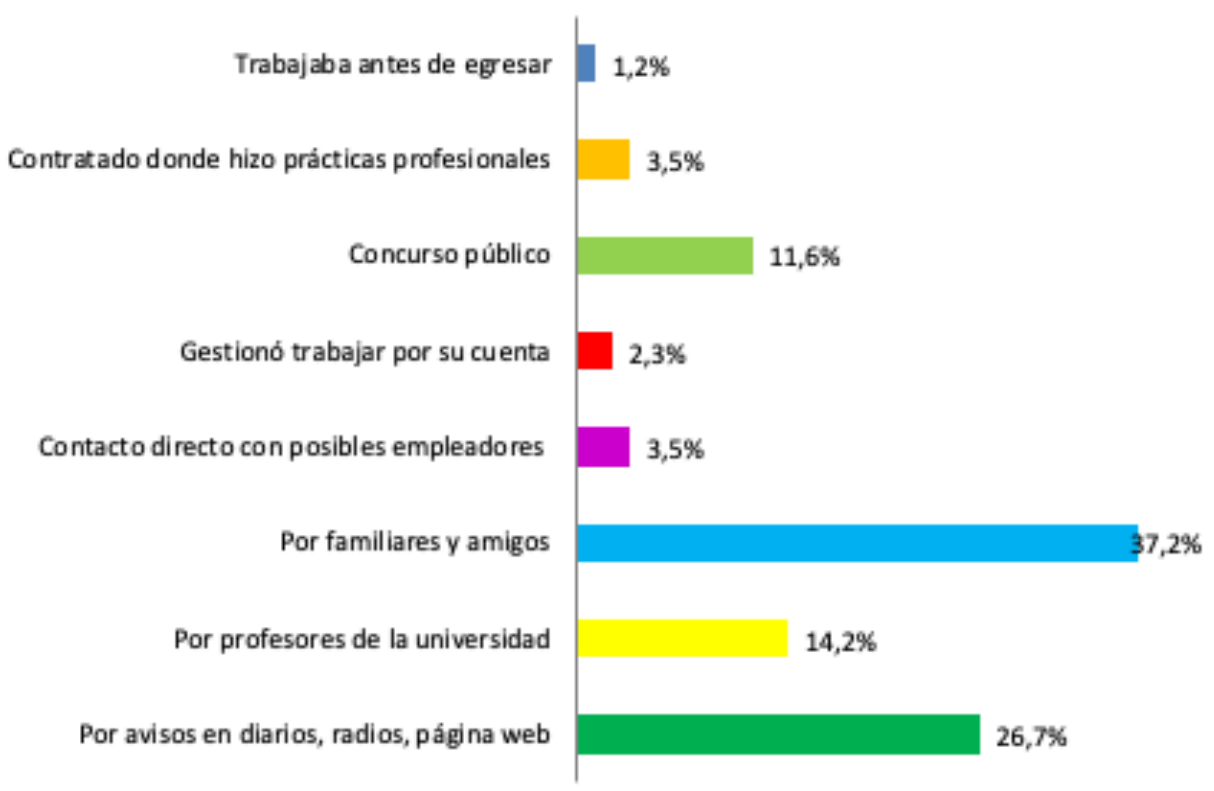

Gráfico 2. Forma de conseguir el primer empleo 
Además de encontrar una alta proporción de egresados con empleo, la mayoría se encontraba desempeñándose en su profesión. Sin embargo, para el ejercicio profesional es requisito la habilitación profesional que confiere el colegio profesional (18), aspecto no considerado en la encuesta. Se reportó que más de la mitad de los colegiados en Lima se encontraba trabajando como dentista antes de su colegiatura, condición calificada como ejercicio ilegal de la profesión (19).

Sobresale el hecho que la mayor proporción de egresados tenga como ocupación secundaria a la actividad odontológica. Esto puede explicarse debido a que la pregunta fue "Además de su ocupación principal la semana pasada, ¿tuvo usted otro trabajo para obtener ingresos?" Es posible que, en su mayoría, se desempeñen profesionalmente en dos lugares.

La inserción laboral de los egresados universitarios oscila el $95 \%$, entre los años 2012 y 2016 , cifra que no difiere significativamente de la registrada para la población económicamente activa (PEA) en el país. Sin embargo, el empleo formal entre los egresados universitarios se ha sostenido en esos años entre $67 \%$ y el $72 \%$, tasa que casi triplica la correspondiente a la PEA ocupada total. Ello indicaría que una mayor productividad del trabajador, asociada a su mayor nivel educativo, se encontraría relacionada con el estar empleado en condiciones de formalidad (20). El 20\% de los egresados de odontología del estudio que no se encontraban trabajando, es cinco veces más al 4,2\% de los egresados de ciencias de la salud reportado (20). Similar proporción a la nuestra es encontrada en egresados de odontología de una universidad colombiana, atribuyéndose esta situación a una sobreoferta laboral de odontólogos por el aumento de la creación de facultades de odontología (21).

Encontramos un promedio inferior a la jornada laboral de 40 horas, similar a lo reportado en egresados de Colombia con igual tiempo de egreso al del estudio, 34,8 + 14, 5 horas (21). Sin embargo, el promedio de horas trabajadas para los egresados de ciencias de la salud es de 43,7 (20), que puede ser explicado por el tiempo de egreso de apenas tres años en los odontólogos de nuestro estudio. Nuestro resultado podría ser tipificado como sub empleo, puesto que más de la tercera parte de los encuestados manifestó su deseo de trabajar más horas.
El ingreso promedio mensual en el estudio fue aproximadamente la quinta parte de lo reportado, 2611 soles para egresados de ciencias de la salud (20) y la cuarta parte de lo publicado en el portal web Ponte en carrera del Ministerio de Trabajo, 1 954 soles (22). Si bien las cifras no son comparables por la temporalidad en la que fueron registradas, mostrarían un ingreso menor en los egresados de esta investigación. Por otro lado, es posible que preguntar sobre el ingreso percibido esté generando rechazo y los egresados hayan respondido una cifra inferior a la real por evitar pago de impuestos o por propia seguridad. Por ello, este dato pude ser discutible.

Más allá de la reconocidaimportancia delainserción laboral de los egresados como una dimensión clave para medir los resultados del proceso universitario, condiciones externas a la universidad tales como el capital social, los antecedentes familiares, etnicidad y el sexo del egresado han evidenciado ser también determinantes significativos del éxito laboral medido en términos de remuneración, adecuación laboral o formalidad. Además, existen habilidades no cognitivas tales como emprendedurismo, autodeterminación y resiliencia, entre otras, que influyen también en las condiciones laborales de los egresados (20). Un reporte en egresados de odontología de una universidad pública (23), encuentra que los factores asociados a la inserción profesional fueron los sociodemográficos (lugar de residencia: Perú o el extranjero) y los factores educacionales relacionados al pregrado (haber realizado ayudantías y el estudio de idiomas culminados). Estos factores no fueron considerados en nuestra investigación.

El hecho que la tercera parte de los egresados procedan de Lima estaría respondiendo a que la mitad de los casi 45 mil odontólogos del país, ejerce en Lima. Esta centralización en la capital estaría provocando una sobreoferta odontológica, y por el contrario, en algunas de las regiones del resto del país, ocurriría una escasez de profesionales. Esta distribución inadecuada de los profesionales de salud podría impactar en la salud bucal de la población y en la situación laboral de los profesionales.

Un aspecto positivo es que, descontando al aproximadamente $40 \%$ que egresó en el año previo al estudio, casi la totalidad de los participantes del estudio se encontraban titulados. Además, la tercera parte 
había realizado o se encontraba realizando estudios de posgrado como diplomados, lo cual implica un perfeccionamiento continuo que incrementa las probabilidades de mayor empleabilidad.

Aspectos como el encontrarse laborando, que la ocupación secundaria sea en el área profesional, el corto tiempo que demandó buscar el primer empleo y el número de empleos que obtuvieron, indicaría que los egresados adquirieron competencias para la empleabilidad.

\section{Correspondencia:}

Milagros Bertha Carrasco Loyola

Correo electrónico: milagros.carrasco@upch.pe

\section{REFERENCIAS BIBLIOGRÁFICAS}

1. Campos G. Implicaciones del concepto de empleabilidad en la Reforma Educativa. Revista Iberoamericana de Educación. 2003.

2. Campos G. Implicaciones económicas del concepto de empleabilidad. Aportes. 2003;8(23):101-111. (Fecha de acceso: 18 de abril del 2019). Disponible en: http:// www.redalyc.org/pdf/376/37602308.pdf

3. Suárez B. Empleabilidad: análisis del concepto. Revista de Investigación en Educación. 2016;14(1):6784. (Fecha de acceso: 18 de abril del 2019). Disponible en http://reined.webs.uvigo.es/ojs/index.php/reined/ article/view/1140/376

4. Real Academia Española. Empleabilidad. Madrid: Real Academia Española; 2014. (Fecha de acceso: 18 de abril del 2019). Disponible en https://dle.rae.es/ empleabilidad? $\mathrm{m}=$ form

5. Hillage J, Pollard E. Employability: Developing a framework for policy analysis. Labour Market Trends. 1998;107:83-84. (Fecha de acceso: 18 de abril del 2019). Disponible en: https://www.researchgate.net/ publication/225083565_Employability_ Developing_a_framework_for_policy_analysis

6. Weinberg P. Formación profesional, empleo y empleabilidad. 2004. En: Formichella MM, London S. Empleabilidad, educación y equidad social. Revista de Estudios Sociales. 2013;47:79-91. (Fecha de acceso: 18 de abril del 2019). Disponible en: http://www. redalyc.org/html/815/81529190007/

7. Yorke M. Employability in higher education; what it is what it is not. The Higher Education Academy. 2006; Serie uno Learning and employability. (Fecha de acceso: 18 de abril del 2019). Disponible en: https://www.researchgate.net/publication/225083582 Employability_in_Higher_Education_What_It_Is_What_It_Is_Not
8. Martínez J. La empleabilidad: una competencia personal y una responsabilidad social. Contribuciones a las Ciencias Sociales. 2011. (Fecha de acceso: 18 de abril del 2019). Disponible en: www.eumed.net/rev/ $\operatorname{cccss} / 15 /$

9. Argote L, Duque A, Gonzales L, Payán C, Rojas L. Los egresados y su desempeño en el medio; un desafío de las instituciones formadoras del recurso humano en salud. Colom Med. 2001;34(4):169-173.

10. Sistema Nacional de Evaluación, Acreditación y Certificación de la Calidad Educativa. Modelo de acreditación para programas de estudios de educación superior universitaria. 2017. (Fecha de acceso: 18 de abril del 2019). Disponible en: https://www.sineace. gob.pe/wp-content/uploads/2014/08/Anexo-1-nuevomodelo-programas-Resolucion-175.pdf

11. Teichler U. La importancia de la educación superior. Conclusiones del estudio CHEERS. En: Mora JG, Schomburg H, Teichler U. Empleo y trabajo de los graduados universitarios. Conclusiones de diferentes estudios. 2013. Barcelona: Ediciones Octaedro, S.L.

12. Schomburg H. El trabajo profesional de los graduados. En: Mora JG, Schomburg H, Teichler U. Empleo y trabajo de los graduados universitarios: Conclusiones de diferentes estudios. Barcelona: Ediciones Octaedro SL; 2013.

13. Carot JM, Conchado A, Mora JG. PROFLEX: un sistema de seguimiento de graduados para Latinoamérica. En: Mora JG, Schomburg H, Teichler U. Empleo y trabajo de los graduados universitarios. Conclusiones de diferentes estudios. 2013. Barcelona: Ediciones Octaedro SL.

14. Vélez J, Oquendo C, Puello P. Perfil del egresado de pregrado de la facultad de odontología del CES 20002004. Revista CES Odontología. 2008;21(1);27-32.

15. Webb L. Perfil ocupacional del egresado de la Facultad de Estomatología de la Universidad Peruana Cayetano Heredia, entre los años 1975 y 1999. Tesis Mg en Estomatología. Lima, Perú: Universidad Peruana Cayetano Heredia; 2002.

16. INEI. Encuesta Nacional a Egresados Universitarios y Universidades, 2014. Lima: INEI; 2015. (Fecha de acceso: 18 de abril del 2019). Disponible en: https:// www.inei.gob.pe/media/MenuRecursivo/ publicaciones_digitales/Est/Lib1298/Libro.pdf

17. Ministerio de Trabajo y Promoción de Empleo. Informe anual del empleo en el Perú.Lima: Ministerio de Trabajo y Promoción de Empleo; 2014. (Fecha de acceso: 18 de abril del 2019). Disponible en: https:// www.trabajo.gob.pe/archivos/file/estadisticas/peel/ enaho/INFORME_ANUAL_EMPLEO_ ENAHO_2014.pdf (Fecha de acceso: 18 de abril del 2019).

18. Congreso de la República. Reglamento de la Ley $\mathrm{N}^{\circ}$ 29016. Lima: Diario El Peruano; 2008. (Fecha de 
acceso: 18 de abril del 2019). Disponible en: http:// www.cop.org.pe/pdf/L_Reglamento_Ley_29016 Ley_que_modifica_articulos_de_la_Ley_15251.pdf

19. Colegio Odontológico del Perú. Apuntes sobre el ejercicio de la odontología en el Perú. Situación laboral previa de nuevos colegiados procedentes de universidades nacionales y privadas de Lima (Ejercicio ilegal de la odontología en Lima). Lima: Colegio Odontológico del Perú; 2012. (Fecha de acceso: 18 de abril del 2019). Disponible en: http:/www.cop.org.pe/ pdf/LIBRO_Apuntes_Ejercicio_Odontologia_2013. pdf

20. Superintendencia Nacional de Educación Superior Universitaria. Informe Bienal sobre la realidad universitaria peruana. Lima: Superintendencia Nacional de Educación Superior Universitaria ;2018. (Fecha de acceso: 18 de abril del 2019). Disponible en: https://www.sunedu.gob.pe/informe-bienal-sobrerealidad-universitaria/

21. Arroyo M, Chia K, González F. Situación laboral de los odontólogos generales de la Universidad Santo Tomás registrados en la Base de Datos de Egresados durante el periodo de 1992-2017. Tesis de grado para optar el Título de Odontólogo. Bucaramanga,
Colombia: Facultad de Odontología, Universidad de Bucaramanga; 2018. (Fecha de acceso: 18 de abril del 2019). Disponible en: http://repository.usta.edu.co/bitstream/handle/ 11634/11632/2018mariaarroyokaterinchia.pdf? sequence $=1$

22. Ministerio de Trabajo y Promoción de Empleo. Plataforma Ponte en carrera, Perú.Lima: Ministerio de Trabajo y Promoción de Empleo; 2018. (Fecha de acceso: 18 de abril del 2019). Disponible en: https:// www.ponteencarrera.pe/como-va-el-empleo\#cuantoganan

23. Valle L. Factores asociados a la inserción profesional del egresado de la Facultad de Odontología de la Universidad Nacional Mayor de San Marcos. Tesis para obtener el grado de Magíster en Estomatología. Lima,Perú:Universidad Nacional Mayorde San Marcos; 2012. (Fecha de acceso: 18 de abril del 2019). Disponible en: http://cybertesis.unmsm.edu.pe/bitstream/handle/ cybertesis/4403/Valle_rl.pdf? sequence $=1$ \&isAllowed $=y$ (Fecha de acceso: 18 de abril del 2019).

Recibido : 15-07-2020

Aceptado : 14-12-2020 